\title{
Ultrasound-guided radial nerve block to relieve cannulation- induced radial arterial spasm
}

\author{
Pradipta Bhakta, MD, FCAI, EDRA (1) - Humayun Zaheer, MD
}

Received: 2 April 2017/Revised: 26 May 2017/Accepted: 7 August 2017/Published online: 10 August 2017

(C) Canadian Anesthesiologists' Society 2017

\section{To the Editor,}

We report our experience using ultrasound-guided radial nerve block to relieve radial artery spasm (RAS) in two patients after cannulation of the radial artery. Case 1 was a 76-yr-old man admitted to our intensive care (ICU) in acute respiratory failure two weeks after repair of an open abdominal aortic aneurysm. Comorbidities included smoking and alcohol abuse, hypertension, hypercholesterolemia, congestive cardiac failure, coronary artery disease, and chronic obstructive pulmonary disease. Case 2 was a 75 -yr-old woman admitted to the ICU with an acute sigmoid diverticulitis complicated by a subphrenic abscess. Comorbidities included a history of smoking, hypertension, and Alzheimer's disease.

In both patients, the radial artery was cannulated just proximal to the wrist crease using a 20G Teflon-coated cannula (B. Braun Medical Inc., Melsungen, Germany). The arterial cannulations were technically difficult and required more than one attempt. Following cannulation, a normal arterial waveform was obtained, the arterial line flushed easily, and blood was freely aspirated. After functioning well for about four hours, the arterial waveform began to dampen in each patient, gradually disappearing over a two-hour interval along with the ability to aspirate blood. After ruling out mechanical causes (e.g., kinks, air bubbles) various maneuvers were tried, including

This letter is accompanied by an editorial. Please see Can J Anesth 2017; 64: this issue.

P. Bhakta, MD, FCAI, EDRA $(\bowtie) \cdot$ H. Zaheer, MD

Department of Anesthesia and Intensive Care, University

Hospital Kerry, Tralee, Ireland

e-mail: bhaktadr@hotmail.com withdrawing and repositioning the catheter, vigorous line flushing with saline, and local application of heat to the hand and forearm - all to no avail. Ultrasonography confirmed that the catheter was correctly positioned in the artery. Unexpectedly, the artery appeared to be nonpulsatile from the cannulation site to about $5 \mathrm{~cm}$ below the elbow crease, and a pulse was not palpable over this distance. For both patients, the non-invasive mean arterial blood pressure measured on the contralateral arm was 65$75 \mathrm{mmHg}$.

We hypothesized that the problems with the radial arterial line were secondary to RAS, and a radial nerve block was performed as a rescue measure. We assumed that sympathetic postganglionic axons accompanying the radial nerve would be blocked and that the diminution of sympathetic tone would help reverse the arterial spasm. Under ultrasound guidance, radial nerve block was achieved by injecting $5 \mathrm{~mL}$ of $0.5 \%$ levobupivacaine with a 22G Sonoplex needle (Pajunk, Norcross, GA, USA) about $5 \mathrm{~cm}$ distal to the elbow crease, just lateral to the radial artery where pulsatile arterial movement was visible. Within $30 \mathrm{~min}$ of injection, an arterial waveform reappeared. In addition, although damped in appearance, blood could be aspirated. After about one hour, the waveform assumed a normal appearance, and the arterial pressure measurements were similar to those of the noninvasive readings, suggesting reversal of an arterial spasm.

The radial artery is a favoured site for invasive monitoring of arterial pressure because of its accessibility, ease of cannulation, and relatively low risk of complications. ${ }^{1}$ Transient RAS, thought to be secondary to trauma of the arterial wall, has been typically associated with trans-radial percutaneous coronary interventions using wider-bore (i.e., 14G) catheters. In this setting, RAS has been reported to be as high as $57 \% .^{2}$ Presumably, arterial 
trauma is reduced with smaller-gauge catheters, although RAS may still be observed. ${ }^{2}$ The underlying mechanism of RAS remains to be fully elucidated, although activation of $\alpha$-adrenergic receptors in arterial smooth muscles has been implicated. ${ }^{2}$ Peripheral vascular disease, hypertension, traumatic catheter insertion, local reaction to the catheter material, and prolonged catheter insertion are risk factors for RAS. ${ }^{2,3}$

Radial artery spasm interferes with patient monitoring and can lead to limb ischemia. ${ }^{1,2}$ Measures used to relieve RAS include local warming, intra-arterial injection of lidocaine and vasodilators (calcium channel blockers, nitroglycerine), and sympatholytic block (stellate ganglion and brachial plexus block). ${ }^{1-5}$ Each of these remedies has drawbacks. For example, local warming may be ineffective, and intra-arterial vasodilators may precipitate hypotension. Central sympathetic blocks may be technically challenging and result in excessive sympatholysis. In contrast, radial nerve block may offer a safe, simple, effective solution.

Conflicts of interest The authors report no conflicts of interest, and no authors or their relatives have connections with anyone or with pharmaceutical companies that could have implications regarding this case report.
Editorial responsibility This submission was handled by Dr. Steven Backman, Associate Editor, Canadian Journal of Anesthesia.

Funding No funding was requested for the study. We declare that we have no financial and/or personal relationships with other people or organizations that could inappropriately influence.

\section{References}

1. Scheer BV, Perel A, Pfeiffer UJ. Clinical review: complications and risk factors of peripheral arterial catheters used for haemodynamic monitoring in anaesthesia and intensive care medicine. Crit Care 2002; 6: 199-204.

2. Brzezinski M, Luisetti T, London MJ. Radial artery cannulation: a comprehensive review of recent anatomic and physiologic investigations. Anesth Analg 2009; 109: 1763-81.

3. Wallach $S G$. Cannulation injury of the radial artery: diagnosis and treatment algorithm. Am J Crit Care 2004; 13: 315-9.

4. Soni KD, Sawhney C, Kaur M, Ramchandani S, Singhal M. Stellate ganglion block as a limb salvaging technique. Indian $\mathbf{J}$ Anaesth 2012; 56: 307-8.

5. Breschan $C$, Kraschl R, Jost $R$, Marhofer P, Likar $R$. Axillary brachial plexus block for treatment of severe forearm ischemia after arterial cannulation in an extremely low birth-weight infant. Paediatr Anesth 2004; 14: 681-4. 